COLO-HEP-469

hep-th/0106174

\title{
Moduli stabilization and supersymmetry breaking in effective theories of strings
}

\author{
R. Brustein ${ }^{(1)}$ and S. P. de Alwis ${ }^{(2)}$ \\ (1) Department of Physics, Ben-Gurion University, Beer-Sheva 84105, Israel \\ (2) Department of Physics, Box 390, University of Colorado, Boulder, CO 80309. \\ e-mail: (1) ramyb@bgumail.bgu.ac.il, (2)dealwis@pizero.colorado.edu
}

\begin{abstract}
The region of moduli space of string theories which is most likely to describe the "real world" is where the string coupling is about unity and the volume of extra compact dimensions is about the same size as the string volume. Here we map the landscape of this "central" region in a model-independent way, assuming only that the string coupling and compact volume moduli are chiral superfields of $\mathcal{N}=1$ supergravity (SUGRA) in 4 dimensions, and requiring only widely accepted conditions: that the supersymmetry (SUSY) breaking scale is about the weak scale and that the cosmological constant be of acceptably small magnitude. We find that the superpotential has (in the supersymmetric limit) a fourth order zero in the SUSY breaking direction. The potential near the minimum is very steep in the SUSY preserving directions, and very flat in the SUSY breaking direction, consequently the SUSY breaking field has a weak scale mass, while other moduli are heavy. We also argue that there will be additional near by minima with a large negative cosmological constant.
\end{abstract}


The most popular solution to the gauge hierarchy problem, i.e. the stability of the tiny ratio $m_{W} / M_{P} \sim 10^{-16}$ between the weak scale and the Planck scale, envisages a $\mathcal{N}=1$ SUGRA broken by some non-perturbative field theoretic effects in some "hidden" sector [1]. In this context it is commonly held that one should compactify string theory on Ricci flat manifolds such as a Calabi-Yau (CY) spaces. Alternatively, in some brane world scenarios the hidden sector lives on one of the branes and SUSY breaking is transmitted by gravitational effects to the "visible" brane, on which our universe lives. However, the potentials that are generated for the dilaton and the compactification moduli are typically of the runaway form [2] so that the theory prefers to go to the zero coupling and decompactification limit. Similar problems are encountered in theories in which SUSY is broken completely by string theoretic effects, such as the Scherk-Schwarz mechanism where the compactification explicitly breaks SUSY.

If one tries to stabilize moduli by introducing a more complicated hidden sector as in race-track models [3], then SUSY is broken at some intermediate scale (about $10^{13} \mathrm{GeV}$ ). Alternatively, if one uses string theoretic mechanisms, such as the Scherk-Schwarz mechanism with the radius of compactification stabilized by quantum effects, or models with $\mathrm{D}$ branes and anti D branes at some orbifold fixed points or D-branes at angles, SUSY is broken at the string scale. In such mechanisms one invariably encounters what we have called the "practical cosmological constant problem" (PCCP) [ [ that to a given accuracy within a given model the cosmological constant vanishes. This is equivalent to the requirement that models should at the very least allow for the possibility of a large universe to exist with reasonable probability. This is not the same as requiring a solution to the "cosmological constant problem" [5]: why is the cosmological constant so small in natural units? [6]

Thus, currently available stringy SUSY breaking and moduli stabilization mechanisms are simply not viable. They do not even allow a large universe, let alone finding an explanation for one. One could take the point of view that the difficulties are "technical", and that they will eventually be resolved when computational technology improves, and 
therefore simply ignore them. We believe that the difficulties are not technical, but rather require some changes in the basic framework. Here we show what modifications are needed in the input from string theory and how they can improve the situation significantly. We will not have anything to say about the precise string mechanisms that may lead to these modifications. However, the point is that if string theory is to solve even the PCCP, such a mechanism would have to be found.

A generic resolution of the PCCP requires a continuously adjustable constant in the potential. Consequently, in a $\mathcal{N}=1$ SUGRA such a constant in the superpotential is required. So what could be its origin? There is no mechanism known to us for its generation from field theoretic effects, so if it exists, it must originate directly from string theory. General solutions to the string equations of motion have integration constants, but so far only restricted choices of these constants have been made. In the standard string phenomenology based on $\mathrm{CY}$, or $D \bar{D}$ systems there is no room for a continuously adjustable constant in the superpotential. It is the Ricci flatness condition on CY (or orbifold) compactfications that amounts to a restriction that overly constrains the 4D moduli potential. On the other hand brane world scenarios with compactification on (squashed) spheres, such as type IIB on a squashed 5-sphere with $D_{3}$ branes [7] allow a less restrictive framework, with room for an adjustable constant. In particular, it is possible to choose integration constants to get flat brane solutions after SUSY breaking [8]. However it is still unclear whether such models, which so far have only been constructed within IIB supergravity, have a string theoretic ultra-violet completion.

Dualities, and the proposed unity of all string theories and 11D SUGRA offer some new perspectives on the problem of moduli stabilization and SUSY breaking. One of the well known lessons of string dualities is that the strong coupling limit of one type of perturbative string theory is another type of perturbative theory (S-duality). Similarly a compactification on a 'small' (compared to the string scale) manifold is dual to a different (or sometimes the same) perturbative theory compactified on a "large" manifold. Now, even in the absence of SUSY breaking one would expect string theoretic non-perturbative (SNP) effects coming 
from the various brane instantons $4,9,10]$. To the extent that their effects can be estimated (and this can only be done in the various weak coupling limits) they give runaway potentials which tend to take the theory to the extreme weak or strong coupling limit and or the extreme decompactification limit. For example, in the case of the S-duality between the heterotic $E_{8} \times E_{8}(\mathrm{HE})$ theory and its strong coupling version the Horava-Witten (HW) theory, the non-perturbative effects due to the F string and the NS fivebrane instantons tend to give a runaway dilaton potential which makes the dilaton roll down to weak coupling whereas in the HW theory the corresponding dual effect originates from the $M$ twobrane and the M fivebrane instantons which tend to push the theory towards infinite radius for the eleventh dimension i.e. to strong coupling. A realistic phenomenolgy would thus require that these effects are merely manifestations of the fact that the investigations are done in the outer regions of moduli space and that in the central region there exists an actual minimum of the potentials for the various moduli [4]. This is the basic idea in the concept of "string universality". Furthermore, we have pointed out, in this context, that the stabilization of the moduli was most probably a string non-perturbative (SNP) effect while SUSY breaking could well be a field theoretic phenomenon.

The scenario that we propose for moduli stabilization and SUSY breaking is then the following. The central region of moduli space is parameterized by chiral superfields of $\mathrm{D}=4$, $\mathcal{N}=1$ SUGRA. They are all stabilized at the string scale by SNP effects which allow a continuously adjustable constant in the superpotential. SUSY is broken at an intermediate scale by field theoretic effects that shift the stabilized moduli only by a small amount from their unbroken minima. The cosmological constant can be made to vanish after SUSY breaking by the adjustable constant. We find that models of such a scenario provide a surprisingly rich central region landscape. Although our motivation for proposing this scenario originates from string universality, our analysis and conclusions are valid whenever a string theory can be approximated by an effective $\mathcal{N}=1$ SUGRA in the central region of moduli space.

We model the central region using two moduli, one parameterizes the SUSY breaking 
direction, and the other is a representative of the orthogonal directions. Keeping with tradition we name them $S$ and $T$, but they are not necessarily the dilaton and volume moduli of any of the perturbative string theories, rather, we expect them to be functions of them and the other moduli (such as shape moduli). We first study stabilization of moduli with unbroken SUSY, as our scenario calls for, but instead of considering the strict limit of unbroken SUSY, we introduce the SUSY breaking scale $\varepsilon \equiv m_{3 / 2} \sim 10^{-16}$ (We use units in which the Planck mass $M_{P}=1$ ), and consider the dependence of various quantities on $\varepsilon$.

The basic requirement is that the "would be" SUSY breaking direction $S$ as well as the orthogonal direction(s) $T$ are stabilized by the SNP superpotential $W_{S N P}$ with unbroken SUSY in the central region at some point $\left(\widetilde{S}_{0}, \widetilde{T}_{0}\right)$. When the field theoretic SUSY breaking is turned on, $\left(\widetilde{S}_{0}, \widetilde{T}_{0}\right)$ will be shifted by terms of $O(\epsilon)$ to $\left(S_{0}, T_{0}\right)$ [11] and should satisfy the following criteria:

(a) a minimum of the potential at $\left(S_{0}, T_{0}\right):\left.\partial_{S} V\right|_{\min }=0,\left.\partial_{T} V\right|_{\min }=0$

(b) with broken SUSY: $\left.F_{S}\right|_{\min } \sim 0(\varepsilon)$

(c) and a small cosmological constant: $\left.V\right|_{\min }<0\left(\varepsilon^{2}\right)$

(d) in the central region: $\operatorname{Re} S_{0}, \operatorname{Re} T_{0} \sim 1$.

In addition, for the SUSY preserving direction $T$ we have

(e) $\left.F_{T}\right|_{\min }=0$.

In the above $\left.\right|_{\min }$ means that the expression is to be evaluated at the minimum of the potential. Similar conditions apply for the complex conjugate quantities.

The potential is given in terms of the Kahler potential $K=K(S, \bar{S}, T, \bar{T})$, and the superpotential $W=W(S, T)$,

$$
V=e^{-K}\left(F_{i} K^{i \bar{j}} F_{\bar{j}}-3|W|^{2}\right)
$$

and the $F$ terms are given by

$$
F_{S}=\partial_{S} W+K_{S} W ; F_{T}=\partial_{T} W+K_{T} W
$$

$K$ with subscripts denote derivatives of the Kahler potential, and $K^{i \bar{j}}$ is the inverse of the 
matrix of second derivatives of the Kahler potential. At the minimum the Kahler metric is taken to be diagonal so that in particular

$$
\left.K^{S \bar{T}}\right|_{\text {min }}=0 .
$$

We will now show that in order to eventually obtain parametrically small $O(\varepsilon)$ SUSY breaking the superpotential and its first three derivatives must be of order $\varepsilon$ if the Kahler potential and its derivatives at the minimum are of order unity, as expected. Conditions (b), (c) and (e) and the definition (2) imply that

$$
\left.W\right|_{\min },\left.\partial_{S} W\right|_{\min },\left.\partial_{T} W\right|_{\min } \sim O(\varepsilon) .
$$

Note also that $\left.\partial \frac{n}{S} F_{S}\right|_{\min }=\left.\partial \frac{n}{S} K_{S} W\right|_{\min } \sim O(\varepsilon)$, and $\left.\partial \frac{n}{S} F_{T}\right|_{\min }=\left.\partial \frac{n}{S} K_{T} W\right|_{\min } \sim O(\varepsilon)$, for $n=$ $0,1,2, \ldots$ Conditions (a),(b),(e) and eq.(3) then implies that $\partial_{S} F_{S} \partial_{S} K^{S \bar{S}} \bar{F}_{\bar{S}}+O\left(\varepsilon^{2}\right)=0$, so that using (2) again we find that $\partial_{S} F_{S} \sim O(\varepsilon)$ and hence $\partial_{S}^{2} W \mid \sim O(\varepsilon)$. Also from (4) and the second equation of (a) we have $\left.\partial_{T} V\right|_{\min }=e^{K} \partial_{T} F_{S} K^{S \bar{S}} F_{\bar{S}}+O\left(\varepsilon^{2}\right)=0$ leading to the estimate

$$
\left.\partial_{T} F_{S}\right|_{\min },\left.\partial_{S} \partial_{T} W\right|_{\min },\left.\partial_{S} F_{T}\right|_{\min } \sim O(\varepsilon)
$$

Now, let us look for conditions on the second derivatives of the potential. Using conditions (a)-(e) and the above estimates we have,

$$
\left.\partial_{S}^{2} V\right|_{\min }=e^{K}\left[\left.\partial_{S}^{2} F_{S} K^{S \bar{S}} \bar{F} \bar{S}\right|_{\min }+O\left(\varepsilon^{2}\right)\right],
$$

so a priori $\left.\partial_{S}^{2} V\right|_{\min } \sim O(\varepsilon)$. On the other hand using our previous estimates we find that $\left.\partial_{S \bar{S}} V\right|_{\min } \sim O\left(\varepsilon^{2}\right)$. Thus, the subdeterminant $H_{S}$ of the matrix of second $S$ derivatives is given at the minimum by

$$
\begin{aligned}
\left.H_{S}\right|_{\min } & =4\left[\left.\left|\partial_{S \bar{S}} V\right|_{\min }\right|^{2}-\left.\left|\partial_{S S} V\right|_{\min }\right|^{2}\right] \\
& =4\left[O\left(\varepsilon^{4}\right)-\left.\left|e^{K} \partial_{S}^{2} F_{S} K^{S} \bar{S} \bar{F} \bar{S}\right|_{\min }\right|^{2}\right] .
\end{aligned}
$$

Now $\left.H_{S}\right|_{\text {min }}$ must be positive. A necessary condition for this is that $\left.\left|e^{K} \partial_{S}^{2} F_{S} K^{S \bar{S}} \bar{F} \bar{S}\right|_{\min }\right|^{2} \sim$ $O\left(\varepsilon^{2}\right)$, which implies that $\partial_{S}^{2} F_{S} \sim O(\varepsilon)$, and therefore that $\left.\partial_{S}^{3} W\right|_{\min } \sim O(\varepsilon)$. 
Second $T$ derivatives are given by

$$
\begin{aligned}
\left.\partial_{T \bar{T}} V\right|_{\min } & =e^{K} \partial_{T} F_{T} K^{T \bar{T}} \partial_{\bar{T}} \bar{F}_{\bar{T}}+O(\varepsilon) \\
\left.\partial_{T}^{2} V\right|_{\min } & =e^{K} 2 \partial_{T} F_{T} K^{T \bar{T}} \partial_{T} \bar{F}_{\bar{T}}+O(\varepsilon) .
\end{aligned}
$$

But since $\partial_{T} \bar{F}_{\bar{T}}=\partial_{T} K_{T} \bar{W} \sim O(\varepsilon)$, it follows that $\left.\partial_{T}^{2} V\right|_{\min }$ is $O(\varepsilon)$. Therefore the subdeterminant $H_{T}$ of $T$ second derivatives $H_{T}=4\left[\left.\left|\partial_{T} V\right|_{\min }\right|^{2}-\left.\left|\partial_{T T} V\right|_{\min }\right|^{2}\right]$ can be positive with $\partial_{T} F_{T}=O(1)$. This implies that (generically) $\partial_{T}^{2} W \sim O(1)$, and that $\left.\partial_{T \bar{T}}^{2} V\right|_{\min } \sim O(1)$.

Summarizing our results so far we find that the superpotential at the minimum must satisfy

$$
\begin{aligned}
& \left.W\right|_{\min },\left.\partial_{S} W\right|_{\min },\left.\partial_{T} W\right|_{\min },\left.\partial_{S}^{2} W\right|_{\min }, \\
& \left.\partial_{S T}^{2} W\right|_{\min },\left.\quad \partial_{S}^{3} W\right|_{\min } \sim O(\varepsilon) .
\end{aligned}
$$

On the other hand $\partial_{T}^{2} W$ and $\partial_{S}^{4} W$ and higher derivatives in both $S$ (of order greater than 3 ) and in $T$ (greater than 1), as well as mixed derivatives of order greater than 2 are generically of order unity. In the supersymmetric limit $\varepsilon \rightarrow 0$, the landscape of the central region looks very different in the $S$ and $T$ directions. In the $T$ direction the potential $V$ is very steep, all derivatives from the second derivative and up are generically of order 1 at the minimum. In the $S$ direction the potential is flat around the minimum. In particular the masses of the SUSY breaking $S$ moduli will be of order $\varepsilon$ while in general the masses of the $T$ moduli will be of order one.

We now focus on the SUSY breaking direction $S$. First, at a high scale all moduli are stabilized by SNP, and then at a lower scale SUSY is broken. Our general considerations imply that $W_{S N P}$ has a fourth order zero at the minimum $\widetilde{S}_{0}$. Thus, the simplest model for the SNP superpotential in the SUSY breaking direction is $W_{S N P}=a_{4}\left(S-\widetilde{S}_{0}\right)^{4}$, with $a_{4}, \widetilde{S}_{0} \sim O(1)$. The existence of an adjustable constant is reflected in that the combination $a_{4} \widetilde{S}_{0}^{4}$ is continuously adjustable even though both $\widetilde{S}_{0}$ and $a_{4}$ are of order unity. To realize the separate scale of stabilization of $S$ and of SUSY breaking there should be an additional small field theoretic contribution $\Delta W_{F T}$ (originating, for example, from gaugino condensation) 
to the superpotential. The new SUSY breaking minimum $S_{0}$ of the modified potential is shifted by a small amount from the original minimum $\widetilde{S}_{0}$, and at $S_{0}$ the cosmological constant vanishes (more precisely, it is smaller than $\varepsilon^{2}$ ). We expect that all non-perturbative field theoretic effects are proportional to $e^{-1 / g_{Y M}^{2}}, g_{Y M}$ being the unified coupling of the field theory. $\mathcal{N}=1$ SUGRA prescribes a chiral superfield whose expectation value is $\langle S\rangle=$ $1 / g_{Y M}^{2}$. It follows that the SUSY breaking direction $S$ is in fact well approximated by the very same direction. Note that $S$ could be very different from any perturbative dilaton. In the central region it will be some complicated function of the ten dimensional perturbative dilaton and all the other perturbative moduli of any one theory at a corner of the M-theory moduli space. Also we have not specified what the Kahler potential of the field $S$ should be beyond making the reasonable assumption that it and all its derivatives are of order one.

In summary, we expect additional terms in the superpotential of the form, $\Delta W_{F T}=$ $\sum b_{i} e^{-\beta_{i} S}$. Now, this correction term needs to be $\Delta W \sim O(\varepsilon)$, to generate the hierarchy $m_{3 / 2} / M_{P} \sim 10^{-16} \sim e^{-40}$. This implies that parameters $\beta_{i}$ (which are constants that are determined, for example, by the gauge group of some hidden sector) need to be $\beta_{i} \sim 40$ for prefactors $b_{i} \sim O(1)$. Alternatively, we may expand $\Delta W_{F T}$ around the SUSY breaking minimum, $\Delta W_{F T}=\sum c_{i} e^{-\beta_{i}\left(S-S_{0}\right)}$, so $c_{i} \sim O(\varepsilon)$. In the presence of $\Delta W_{F T}$ the modified superpotential in the central region is of the following form

$$
\begin{aligned}
W= & a_{4}\left(S-S_{0}\right)^{4}+a_{3}\left(S-S_{0}\right)^{3}+ \\
& a_{2}\left(S-S_{0}\right)^{2}+a_{1}\left(S-S_{0}\right)+a_{0}
\end{aligned}
$$

In this expression all coefficients except for $a_{4}$ are of $O(\varepsilon)$. The condition for a vanishing cosmological constant becomes,

$$
a_{1}=\left( \pm \sqrt{3 K_{S \bar{S}}}-K_{S}\right) a_{0}
$$

This is a fine-tuning condition that almost certainly cannot be satisfied if $a_{0}, a_{1}$ originate from the field theoretic term $\Delta W_{F T}$ alone where the relation involves the parameters $b_{i}$ and $\beta_{i}$ which are fixed by the field theory. Therefore, we argue that that $a_{0}$ is a constant coming 
from string theory that is able to adjust itself so as to satisfy (11). Thus, in our model we can take, $a_{4} \sim O(1), a_{3}=-\frac{1}{6} \sum c_{i} \beta_{i}^{3}, a_{2}=\frac{1}{2} \sum c_{i} \beta_{i}^{2}, a_{1}=-\sum c_{i} \beta_{i}$ and $a_{0}$ is an adjustable constant which guarantees (11).

Let us check that there is no obvious obstruction in this model for having a SUSY breaking minimum by looking at the determinant of second S-derivatives and showing that it can be positive. The first term of $H_{S}$ is $\left.\left.\left|\partial_{S \bar{S}} V\right|_{\min }|\sim 4| a_{2}\right|^{2} K^{S \bar{S}}\right|_{\min }=\left.\left(\sum \beta_{i}^{2} c_{i}\right)^{2} K^{S \bar{S}}\right|_{\min }$ while in the second term $\left|\partial_{S \bar{S}} V\right|_{\min } \mid$ the largest component $\left.\sim 6 a_{3} a_{1} K^{S \bar{S}}\right|_{\min }=$ $\left.\left(\sum \beta_{i}^{3} c_{i}\right)\left(\sum \beta_{i} c_{i}\right) K^{S \bar{S}}\right|_{\text {min. }}$. Thus the positivity of $H$ depends on the details of the model. We have analyzed numerically various potentials, and found that there is a range of parameters for which a minimum at $S_{0}$ exists. For example, a minimum at $S_{0}=1$ occurs if $a_{1}=9 a_{3}$, and $a_{2}=-\frac{3-\sqrt{3}}{4} a_{1}$.

The fact that $W_{S N P}$ has a fourth order zero (in the SUSY limit) and therefore that all the coefficients up to and including $a_{3}$ are $O(\epsilon)$ has an unwanted, but generic, consequence: additional minima which have a $O\left(\epsilon^{2}\right)$ negative cosmological constant. Here we show how this comes about. If both $F_{S}$ and $F_{T}$ vanish at some point then the potential is negative, since at this point $V=-3|W|^{2}$. Of course, there is no guarantee that there is a solution to these conditions, however, we can argue reliably that near our SUSY breaking minimum the potential becomes negative. This implies that the minimum with vanishing cosmological constant is not the global minimum. First, note that since we are interested in the neighborhood of the point $\left(S_{0}, T_{0}\right)$ it is enough to keep the terms in $W$ given in (10). Let us fix $T=T_{0}$. Now $F_{S}=\partial_{S} W+K_{S} W \simeq 4 a_{4}\left(S-S_{0}\right)^{3}+O(\varepsilon)$ has zeros at a distance of $\left(S-S_{0}\right) \sim \varepsilon^{1 / 3}$. At this distance $W=W\left(S_{0}\right)+\partial_{S} W_{\min }\left(S-S_{0}\right)+\cdots$ is of order $\varepsilon$, and $F_{T}=\left.\left(S-S_{0}\right) \partial_{S} F_{T}\right|_{\text {min }}+\cdots \sim \epsilon^{4 / 3}$ (see (9)) is of higher order. This means that at such distances the potential is already negative. Thus, the SUSY breaking minimum that we have identified cannot be a global minimum. It is only a local minimum. A full discussion of the properties of these additional minima requires more detailed information on the Kahler potential and the superpotential and will not be attempted here. The appearance of regions of negative potential is generic in $\mathcal{N}=1$ SUGRA theories, and their significance and impli- 
cations should be discussed in a cosmological context. We need to determine whether the fields can move classically to the dangerous negative potential regions, and whether they are stable if they are already in the zero cosmological constant minimum. We will discuss this issue separately.

Once we have obtained the SUSY breaking potential, which, for the purpose of computing soft SUSY breaking terms, could be well approximated by a Polonyi type model with a linear superpotential $W=X+Y\left(S-S_{0}\right)$, we may follow the discussion of 12,13 to obtain soft masses and relations between them. However, to obtain actual spectra we need the coupling of matter fields and gauge fields in the observable sector to moduli in the central region. But these couplings are not available from perturbative calculations and have to be constrained by some general considerations. When (and if) expected results from the Tevatron and LHC determine some of the SUSY soft terms, we may use them to probe couplings in our effective theory.

In conclusion, the assumptions that we made were as follows:

i) The four dimensional low energy effective theory of strings is a $\mathcal{N}=1$ supergravity.

ii) All moduli are stabilized at around $\mathrm{O}(1)$ by stringy non-perturbative effects which generate a superpotential for them in the low energy theory.

iii) The superpotential and the Kahler potential coming from stringy non-perturbative effects have only order 1 terms.

iv) Hierarchically small SUSY breaking effects are generated by field theoretic effects at some intermediate scale.

v) The cosmological constant vanishes (or is small).

We have shown that

I) The superpotential generated by SNP effects must (in the supersymmetric limit) have a fourth order zero in the supersymmetry breaking direction $S$, and needs to have only a second order zero in the SUSY preserving directions $T$.

II) The potential is very steep in the $T$ directions while it is very flat in $S$ directions near the minimum. Consequently, SUSY breaking moduli have electroweak scale masses, while 
in general SUSY preserving moduli will have string scale masses.

III) In order to ensure a vanishing or small cosmological constant there must be an adjustable constant in the superpotential originating directly from string theory that tracks the low energy SUSY breaking effects [14].

\section{ACKNOWLEDGMENTS}

We thank I. Antoniadis, J. Lykken, B. Ovrut and S. Kachru for useful discussions. This research was partly supported by grant No. 1999071 from the United States-Israel Binational Science Foundation (BSF), Jerusalem, Israel. SdA was also supported in part by the United States Department of Energy under grant DE-FG02-91-ER-40672. SdA wishes to acknowledge the hospitality of the ITP Santa Barbara during the final phase of this work. 


\section{REFERENCES}

[1] H. P. Nilles, Phys. Rept. 110, 1 (1984).

[2] M. Dine and N. Seiberg, Phys. Lett. B 162, 299 (1985).

[3] N. V. Krasnikov, Phys. Lett. B193 (1987) 37.

[4] R. Brustein and S. P. de Alwis, "String universality," hep-th/0002087, to be published in Phys. Rev. D.

[5] S. Weinberg, Rev. Mod. Phys. 61, 1 (1989).

[6] The resolution of the cosmological constant problem is extremely important, but is outside the scope of our discussion.

[7] M. S. Bremer, M. J. Duff, H. Lu, C. N. Pope and K. S. Stelle, Nucl. Phys. B543, 321 (1999) hep-th/9807051.

[8] S. P. de Alwis, A. T. Flournoy and N. Irges, JHEP0101, 027 (2001) hep-th/0004125.

[9] E. Lima, B. Ovrut and J. Park, "Five-brane superpotentials in heterotic M-theory," hep-th/0102046.

[10] G. Moore, G. Peradze and N. Saulina, "Instabilities in heterotic M-theory induced by open membrane instantons," hep-th/0012104.

[11] The smallness of the gauge coupling (at unification $g_{Y M}^{2} \sim 1 / 25$ ) is not incompatible with moduli stabilized at $\mathrm{O}(1)$ as discussed in detail in [4].

[12] V. S. Kaplunovsky and J. Louis, Phys. Lett. B 306, 269 (1993) hep-th/9303040.

[13] A. Brignole, L. E. Ibanez and C. Munoz, Nucl. Phys. B 422, 125 (1994) [Erratum-ibid. B 436, 747 (1994)] hep-ph/9308271.

[14] Assuming that there is no hitherto unknown field theoretic mechanism that does this. 